\title{
Immunomodulatory effects of vagus nerve and acetylcholine in gastroduodenal area
}

\author{
Oksana Sulaieva, Natalia Obraztsova \\ Department of Histology, Cytology and Embryology, M. Gorky Donetsk National Medical University, Ukraine
}

Email address:

oksana.sulaieva@gmail.com (O. Sulaieva)

\section{To cite this article:}

Oksana Sulaieva, Natalia Obraztsova. Immunomodulatory Effects of Vagus Nerve and Acetylcholine in Gastroduodenal Area. American Journal of Clinical and Experimental Medicine. Vol. 2, No. 3, 2014, pp. 42-46. doi: 10.11648/j.ajcem.20140203.11

\begin{abstract}
Objective: Neuroimmune interplay is one of the basic elements that determine mucosal health and disease. Continuing discussion of the vagus nerve in maintenance of homeostasis of gastroduodenal area, we aim to revise immunomodulating effects of acetylcholine. Results: Vagus nerve plays a major role in gut mucosa immunity due to cytoprotective properties and modulation of leukocytes activity. Stimulation of vagus nerve after mucosa damage is accompanied by cholinergic anti-inflammatory pathway that is realized directly through alfa7-nicotinergic cholinoreceptors on neutrophils, macrophages and lymphocytes, which activation causes decrease of NF-kB expression and TNF and IL-6 secretion. Indirect effect of acetylcholine through enteroendocrine cells regulation is quite important in determination of neuro-immune-endocrine interplay in gastroduodenal mucosa. In addition, the changes in vagus nerve activity and neurohumoral control of gastroduodenal area under antisecretory therapy of gastroduodenal diseases are deliberated in this review. Conclusion: Vagus nerve dysfunction is likely to be the main cause of gastroduodenal inflammation, dysregeneration and cancerogenesis.
\end{abstract}

Keywords: Gastroduodenal Pathology, Vagus Nerve, Acetylcholine, Immunomodulation

The central nervous system tightly interacts with the immune system to modulate inflammation through humoral and neural pathways. One of the main participant of this cross-talk is acetylcholine (ACh) $[1,2]$. Nowadays it is a well-known fact, that acetylcholine modulates cytokine production in immune cells. This effect involves cells of innate and adaptive immunity and includes direct and indirect mechanisms, which are realized at different types of gastroduodenal pathology. What is the role of vagus nerve and $\mathrm{ACh}$ in the modulation of the immune response? Vagus nerve (VN) has been put forward in the regulation of the "inflammatory reflex", a prototypical reflex circuit that maintains immunological homeostasis [3-4]. The last one involves activation of sensory nerves in spleen under immune response with the consequent stimulation of $\mathrm{VN}$, that also engage the central mechanisms and releasing of acetylcholine in efferent fibers endings of $\mathrm{VN}$ [5].

\section{Cholinergic Anti-Inflammatory Pathway}

In details the chain of events was discovered by
Andersson [4]. First, molecular products of infection or injury activate sensory neurons transmitting signal to the brainstem in vagal centers. Next these incoming signals generate action potentials that travel from the brainstem to the spleen and other organs. All above-mentioned culminates in release of acetylcholine, which can attenuate innate immune responses, modulating activation of the nuclear factor kappa beta (NF-kB) with consequent proinflammatory cytokines production and activation of leukocytes recruitment mechanisms. These effects include not only regulation of monocytes and neutrophils, but also T-lymphocyte-dependent signaling to spleen via VN. This efferent arm of the inflammatory reflex is referred to as the "cholinergic anti-inflammatory pathway".

The most interesting question is how the VN-mediated inflammatory reflex facilitates rapid and specific exchange of information between the nervous and immune systems in order to prevent tissue injury and infection. The cholinergic anti-inflammatory pathway is an efferent vagus nerve-based mechanism that has an anti-TNF-alpha effect both through the release of acetylcholine at the vagal efferents, acting on immune cells, and through the connection of the VN with the spleen, through the splenic sympathetic nerve [6]. 
Protective effect of VN stimulation is connected with modulation of innate immune responses to infection, injury and ischemia through stimulation of alpha7 nicotinic acetylcholine receptors (alpha7nAChR) signaling [7]. Both, $\mathrm{ACh}$ and nicotine, can effectively attenuate macrophage activation by decreasing the production of diverse proinflammatory mediators, including a high mobility group box 1 (HMGB1), TNF-alpha, IL-16, and IL-6. In mouse peritoneal macrophages, LPS-induced TNF-alpha release in vitro was inhibited by a selective alpha7 nAChR agonist, and that effect was attenuated by alpha7 nAChR antagonist methyllycaconitine [3, 8]. In contrast, cholinergic anti-inflammatory pathway was abolished in mice with vagotomy, splenic neurectomy, splenectomy, in alpha7nAChR knockout mice and alpha7nAChR-deficient bone marrow chimaera mice. Besides, the similar results were obtained in Li et al. research [9]. Authors showed that deficiency of alpha7nAChR in bone marrow-derived cells significantly impaired VN-mediated regulation of TNFalpha, whereas alpha7nAChR deficiency in neurons and other cells had no significant effect. It was suggested that the non-neuronal cholinergic system has a regulatory role in inflammation [10].

\section{Alpha7 Nicotinic Acetylcholine Receptors in Maintaining Gastrointestinal Barrier}

Namely alpha-7 nAchR has been shown to be necessary for the VN to modulate the systemic inflammatory response, but the role of alpha- $7 \mathrm{nAchR}$, a ligand-gated ion channel expressed on macrophages, lymphocytes, neurons and other cells, in mediating gut protection remained unknown. It is considered that the main targets of acetylcholine are macrophages and neutrophils expressing alpha7nAChR [8]. Matteoli G. et al. have shown that VN stimulation reduced intestinal inflammation [11]. This effect was associated with inhibition of leukocytes recruitment into the place of gut injury by suppressing the expression of VCAM1. Such effect could be explained by the results of Wallace J.L. [12], who has shown that stimulation of alpha7nAChR on human neutrophils and blood mononuclear phagocytes in vitro attenuates $\mathrm{C} 5 \mathrm{aR}$ - and FcgammaR-triggered generation of reactive oxygen species, expression of leukocyte markers involved in cell recruitment and adhesion, and release of TNF-alpha and other proinflammatory cytokines. The selective 7nAChR agonist GTS21 decreased neutrophils accumulation and release of cytokines and chemokines at sites of injury [13]. Also it is known the ACh inhibitory effect on leukotriene B4 and IL-8 production [12]. Investigation of intracellular mechanisms of this acetylcholine effect has demonstrated that the modulation of intestinal resident macrophages activity via alpha7nAChR was associated with ATP-induced $\mathrm{Ca} 2+$ response $[11,14]$. Stimulation of macrophage $\mathrm{nAChR} \alpha 7$ inhibited nuclear translocation of NF- $\kappa \mathrm{B}$, likely by blocking degradation of the NF- $\kappa$ B inhibitor I $-\kappa \mathrm{B}$ or via activation of signal transducer and activator of transcription STAT3 [2].
This effect resulted in decreased production of IL-1 and TNF, but increased the IL-10 secretion and was realized via microRNA-124, activated under alpha7nAChR stimulation $[15,16]$. Anterograde labeling fails to detect vagal efferents contacting resident macrophages, but shows close contacts between cholinergic myenteric neurons and resident macrophages expressing alpha7nAChR. Instead, the vagus nerve interacts with cholinergic myenteric neurons, which are in close contact with macrophages in GDA [3]. This direct neuro-immune interaction is an alternative for humoral regulation of immunity by the cytokine network [12].

Other cells, including T-lymphocytes, dendritic cells, mast cells, residing in gastric and duodenal mucosa and submucosa express nicotinic receptors and might be affected by the anti-inflammatory pathways as well [15]. RosasBallina $M$. et al. identified an acetylcholine-producing memory phenotype $\mathrm{T}$ cell population in mice that is integral to the inflammatory reflex [7]. The cholinergic CD4(+)CD25(-) lymphocytes were described [17]. These acetylcholine-producing $\mathrm{T}$ cells are required for inhibition of cytokine production by $\mathrm{VN}$ stimulation. One more issue for discussion is $\mathrm{VN}$ impact on dendritic cells maturation and phenotype, which can be implemented by direct and indirect influence through the enteric nervous system and/or enteroendocrine cells [13]. Direct effect of VN via nAChR activation drives immature dendritic cells towards an antiinflammatory phenotype, resulting in TGF- $\beta$ secretion and FoxP3+ regulatory T-cells activation [17]. Interestingly, the same affect was described for vasointestinal peptide secreted by myenteric neurons and endocrine cells [7]. In fact, the vagus activity determines immune homeostasis shifting the balance towards tolerance (normal to enhanced tone) or inflammation (decreased tone).

However it was fond that dendritic cells express not only nicotinic, but muscarinic receptors M3, M4 and M5 too, as well as the enzymes responsible for the synthesis and degradation of $\mathrm{ACh}$, choline acetyltransferase and acetylcholinesterase, respectively. Differentiation of dendritic cells in the presence of the cholinergic agonist carbachol resulted in the stimulation of TNF-alpha and IL-8 production [16]. All these effects were prevented by atropine, a muscarinic $\mathrm{ACh}$ receptor antagonist. Incubation of DC with carbachol after the differentiation increased the expression of HLA-DR, improved the T cell priming ability of dendritic cells, and stimulated the production of TNFalpha, but not IL-12 or IL-10 [13]. Thus the final immunomodulating effect of $\mathrm{VN}$ and Ach depends on target cell receptors expression prevalence.

These facts allow us to reconsider the role of the $\mathrm{VN}$ in regulation of GDA homeostasis. Apparently, the increase of vagal tone could be considered as a compensatory-adaptive reaction, limiting injury, the intensity of inflammation and microcirculatory disturbance [2]. All of the above-mentioned brings up the question: how does medication therapy effect on the neuro-endocrine control and immunological homeostasis in patients with gastroduodenal pathology? 


\section{Effect of Antisecretory Therapy on a Status of Gastric Regulatory Systems}

In recent years the development of new approaches for pharmacological inhibition is rapidly growing up. Owing to the clinical trials, it has been established that there is a direct correlation between mucosal healing and decreasing of acid secretion under the treatment. In this regard, management of acid secretion for a long time has been considered to be one of the most important points.

Historically antisecretory therapy has been started since implementation of antagonists of M-cholinoreceptors. The adoption of M-cholinergic antagonists, once has marked significant progress in the treatment of gastroduodenal pathology. More impressive results were demonstrated for H2-blockers of histamine receptors, although 5-10\% of patients with ulcer disease were resistant to their effects. A detailed study of this phenomenon revealed that M-choline blockers and H2-receptor are blocking only one way of signal transduction to the enzyme, responding for hydrogen ions transport into the stomach's lumen, so that does not exclude other ways for stimulation of acid secretion [18]. This may explain not only the low efficacy of drug's treatment, but also the preservation of acid secretion after a partial resection of the stomach and vagotomy [19].

Development and implementation of proton pump inhibitors (PPIs) into therapeutic practice initially has been considered as a breakthrough in gastroenterology [20]. Emergence of PPIs, which affects on one of the pathogenic mechanisms in ulcer development - peptic factor, it was a new era in solving the problem of acid secretion. PPIs are used both independently and in schemes of eradication therapy, and are considered the "gold standard" in the treatment of acid-related diseases, reducing the level of the hydrochloric acid by $90-100 \%$ (compared with H2-receptor blockers of the third generation, that reduce acid production by $70 \%$ ) [19].

PPIs, linking the protons of hydrogen, interact with the SH-groups of the proton pump, blocking the secretion of hydrochloric acid components in oxyntic cells. However, in addition to a powerful antisecretory effect of PPIs, such kind of therapy also influences on intra- and extraorganic regulatory systems. In a double-blind placebo-controlled study it was shown, that $40 \mathrm{mg}$ of omeprazole daily reduces the motor-evacuation function of the stomach. After 10 days of treatment there was a significant difference in serum concentrations of motilin, gastrin, cholecystokinin. Changing in the stomach's motor function under PPIs is also connected with neural control disorders [20].

At the first step PPIs, inhibiting the hydrochloric acid secretion, activate afferents and vago-vagal reflex, resulting in the release of ACh. However long-term therapy causes disruption of such compensatory mechanisms. A further increase in the synthesis of $\mathrm{ACh}$ is accompanied with increased activity of ACh-esterase that lead to reduction of ACh/AChesterase ratio. This shifting has been observed in patients with frequent recurrent and long-unhealed ulcers [2, 20].
In addition to dysbalance in ACh's regulation of GDA, prolonged use of PPIs and H2-blockers of histamine receptors leads to hyperplasia of ECL- and G-cells, which is considered to be one of the pathogenic mechanisms of recurrent ulceration [21]. The consequence of such reaction of enterochromaffin cells is the increased histamine level and hypergastrinemia [22]. Administration of omeprazole in a standard dose increases gastrin level in 2-3 times. Raising the gastrin level is caused by reduction of hydrochloric acid production and decreased $\mathrm{H}^{+}$level by the feedback mechanism. In this case, stimulation of gastrin secretion develops independently from the activity of vagus efferents.

Increased gastrin level under drug's treatment by itself is a new pathogenic factor in the development of GDA's dysregeneration syndrome. By binding to B-type gastrincholechystokinin receptors, gastrin stimulates hydrochloric acid secretion in parietal cells and activates a number of transcriptional factors that stimulate proto-oncogenes expression [21]. Therefore, hypergastrinemia is accompanied by stimulation of epithelial cell proliferation. And gastrin not only amplifies the physiological cell growth, but can also initiate GDA's mucosal neoplasia. The binding of gastrin or cholecystokinin to receptors is accompanied by activation of a signaling cascade and transduction of mitogenic signal to the nucleus, what results in amplifying of cell proliferation by inducing of non-receptor tyrosine kinases p125fak, Src, p130cas and paksilin. Such enhancement of epithelial cell proliferation could be assessed as a positive point (compensatory mechanism), associated with inflammation [18]. However, interpreting this phenomenon, it is important to remember about the structural and functional denervation (reduced vagal efferent activity), which is closely associated with the myofibroblasts. These cells do not only implement repair, but also control the epithelial cell differentiation and form stem cell niches. Impaired innervation and myofibroblast network arrangement under PPIs administration manifests in gastric mucosa atrophy, and in the development of a neoplastic process. Thus, a drawback of prolonged hypergastrinemia is that there is risk for the development of ESL-cell carcinoid tumors and gastric cancer.

Changes in the gastric mucosa under the PPIs were proved in the clinical trials and confirmed in experiments. Atrophy is characterized by decreased cell renewal with replacing of usual gastric epithelial cells by poorly differentiated cells, not capable of maintaining the mucusbicarbonate barrier [12]. Other one, not least important factor of mucosal atrophy is apoptosis of parietal cells. Reducing of acid secretion by prolonged PPIs use leads to irreversible loss of parietal cells. Here comes up a logical question: how does the reduction of parietal cells correlate with mucosal atrophy of the gastric body? Apparently, this phenomenon is associated with such known trophic and inducing function of parietal cells. The fact listed above corresponds with maintaining of a certain $\mathrm{H}+$ level in the gastric mucosa, as well as with neurotrophic effect due to the stimulation of afferent nerves [20]. Furthermore, it's necessary to emphasize that parietal cells produce a wide 
range of growth factors. These include EGF, HB-EGF, TGF $\beta$, which stimulate cell growth by EGFR activation. Most growth factor receptors are associated with tyrosine kinases that regulate processes of cell proliferation and differentiation. As well, acid secretion by parietal cells is an essential element in maintaining the mucus-bicarbonate barrier on the mucosal surface. In effect, the essence of this phenomenon is hidden in the mechanisms of chloride secretion, transport of chloride ions is going via the basolateral plasmolemma into the cytoplasm of parietal cells in exchange for $\mathrm{HCO} 3$ - anions, catalyzed by carbonic anhydrase. Bicarbonate ions get into the capillaries, which carry the blood up to the surface of mucosa. This circulatory system provides adequate delivery of HCO3-, which is well synchronized with acid production. Therefore, apoptosis and decreased functional activity of parietal cells are associated with limiting of the neurotrophic influences and breakdown of the gastrointestinal barrier [23].

Summarizing all above, it would be necessary to accentuate the role of $\mathrm{VN}$ that it is not just an essential part of digestive physiological control, but also is an indispensable member of GDA compensatory-adaptive processes. Thus, changes of ACh release are accompanied not only with increased motility and secretory activity, but also lead to the following effects: a) increased mucus and bicarbonate production; b) optimization of microcirculation and cytoprotection; c) recovery of the surface epithelium kinetics and connective tissue metabolism; d) restriction of inflammation and secondary alteration of GDA. On the whole these determine the maintenance of the structural and functional organization of the gastro-intestinal barrier. Malfunctions of the brain-gut axis under antisecretory therapy result in inconsistency of neurohumoral regulation of gastric secretion and intercellular cooperation during inflammation.

\section{References}

[1] Ulloa L. The cholinergic anti-inflammatory pathway meets microRNA. Cell Res 2013; 23, №11, 1249-1250.

[2] Sales ME. Cholinergic drugs as therapeutic tools in inflammatory diseases: participation of neuronal and nonneuronal cholinergic systems. Antiinflamm Antiallergy Agents Med Chem 2013; 12, №2, 109-116.

[3] Silva-Herdade AS, Saldanha C. Effects of acetylcholine on an animal mode of inflammation. Clin Hemorheol Microcirc 2013; 53, №1-2, 209-216.

[4] Andersson U, Tracey KJ. Reflex principles of immunological homeostasis. Annu Rev Immunol 2012; 30, 313-335

[5] Bonaz B, Picq C, Sinniger V. et al. Vagus nerve stimulation: from epilepsy to the cholinergic anti-inflammatory pathway. Neurogastroenterol Motil 2013; 25, №3, 208-221.

[6] Huston JM. The vagus nerve and the inflammatory reflex: wandering on a new treatment paradigm for systemic inflammation and sepsis. Surg Infect (Larchmt) 2012; 13, 187-193.

[7] Xiang H, Hu B, Li JG. A new perspective of research into cholinergic anti-inflammatory pathway. Zhongguo Wei Zhong Bing Ji Jiu Yi Xue 2012; 24, №3, 185-188.

[8] Olofsson PS, Katz DA, Rosas-Ballina M. et al. Alpha7 nicotinic acetylcholine receptor (alpha7nAChR) expression in bone marrow-derived non- $\mathrm{T}$ cells is required for the inflammatory reflex. Mol Med 2012; 18, 539-543.

[9] Li J, Mathieu SL, Harris R, et al. Role of alpha7 nicotinic acetylcholine receptors in regulating tumor necrosis factoralpha (TNF-alpha) as revealed by subtype selective agonists. J Neuroimmunol 2011; 239, №1-2, 37-43.

[10] Rosas-Ballina M, Olofsson PS, Ochani M, et al. Acetylcholine-synthesizing $\mathrm{T}$ cells relay neural signals in a vagus nerve circuit. Science 2011; 334, №6052, 98-101.

[11] Matteoli G, Gomez-Pinilla PJ, Nemethova A, et al. A distinct vagal anti-inflammatory pathway modulates intestinal muscularis resident macrophages independent of the spleen. Gut 2013. doi: 10.1136/gutjnl-2013-304676.;

[12] Wallace JL, Ma L. Inflammatory mediators in gastrointestinal defense and injury. ExpBiol Med (Maywood) 2001; 226, №11, 1003-1015.

[13] Vukelic M, Qing X, Redecha P, et al. Cholinergic receptors modulate immune complex-induced inflammation in vitro and in vivo. J Immunol 2013; 191, №4, 1800-1807.

[14] Alsharari SD, Freitas K, Damaj MI. Functional role of alpha7 nicotinic receptor in chronic neuropathic and inflammatory pain: studies in transgenic mice. Biochem Pharmacol 2013; 86, №8, 1201-1207.

[15] Costantini TW, Krzyzaniak M, Cheadle GA, et al. Targeting alpha-7 nicotinic acetylcholine receptor in the enteric nervous system: a cholinergic agonist prevents gut barrier failure after severe burn injury. Am J Pathol 2012; 181, №2, 478-486.

[16] Xu ZP, Devillier P, Xu GN, et al. TNF-alpha-induced CXCL8 production by A549 cells: involvement of the non-neuronal cholinergic system. Pharmacol Res 2013; 68, №1, 16-23.

[17] Pena G, Cai B, Ramos L, et al. Cholinergic regulatory lymphocytes re-establish neuromodulation of innate immune responses in sepsis. J Immunol 2011; 187, №2, 718-725.

[18] Den Hollander WJ, Kuipers EJ. Current pharmacotherapy options for gastritis. Expert Opin Pharmacother 2012; 13, №18, 2625-2636.

[19] Haruma K, Kamada T, Shiotani A. Adverse effects of drugs for peptic ulcer diseases. Nihon Rinsho 2012; 70, Suppl 6, 266-271.

[20] Chubineh S, Birk J. Proton pump inhibitors: the good, the bad, and the unwanted. South Med J 2012; 105, №11, 613618 .

[21] Cui G, Waldum HL. Physiological and clinical significance of enterochromaffin-like cell activation in the regulation of gastric acid secretion. World J of Gastroenterol 2007; 13, № 4, 493-496. 
[22] Zolotarev VA, Khropycheva RP. Effect of proton pump inhibitors on the secretion of bicarbonates and pepsinogen induced by chemical stimulation of the gastric mucosa. Bull Exp Biol Med 2013; 154, №4, 415-418.
[23] Poulsen AH, Christensen S, McLaughlin JK, et al. Proton pump inhibitors and risk of gastric cancer: a populationbased cohort study. Br J Cancer 2009; 100, 1503-1507. 